\title{
Community case management improves use of treatment for childhood diarrhea, malaria and pneumonia in a remote district of Ethiopia
}

\author{
Tedbabe Degefie ${ }^{1}$, David Marsh ${ }^{2}$, Abebe Gebremariam $^{3}$, Worku Tefera ${ }^{4}$, Garth Osborn ${ }^{5}$, Karen Waltensperger ${ }^{6}$
}

\begin{abstract}
Background: Ethiopia's Health Extension Workers (HEW) deliver preventive interventions and treat childhood diarrhea and malaria, but not pneumonia. Most of Ethiopia's annual estimated 4 million childhood pneumonia cases go untreated.

Objective: Evaluate the performance of volunteers in providing Community Case Management (CCM) for diarrhea, fever and pneumonia - in a pre-HEW setting in Liben Woreda, Oromiya Regional State.

Methods: Save the Children supported Ministry of Health and communities to deliver child survival interventions from 1997-2006. We obtained permission in 2005 to train 45 volunteers from remote kebeles in CCM. We evaluated the strategy through reviewing registers and supervision records; examining CCM workers; focus group discussions; and three household surveys.

Results: The CCM workers treated 4787 cases, mainly: malaria (36\%), pneumonia (26\%), conjunctivitis (14\%), and watery diarrhea with some dehydration $(12 \%)$. They saw 2.5 times more cases of childhood fever, pneumonia, and diarrhea than all the woreda's health facility staff combined. Quality of care was good.

Conclusion: The availability, quality, demand, and use of CCM were high. These CCM workers were less educated and less trained than HEWs who perform complicated tasks (Rapid Diagnostic Tests) and dispense expensive antimalarial drugs like Coartem ${ }^{\circledR}$. They should also treat pneumonia with inexpensive drugs like cotrimoxazole to help achieve Millennium Development Goal 4. [Ethiop. J. Health Dev. 2009;23(1):120-126]
\end{abstract}

\begin{abstract}
Background
Ethiopia has an estimated 3,951,000 cases of child pneumonia annually (1), of which 114,000 die (2). Careseeking for cough and difficult or fast breathing is only $19 \%$ (3) which suggests that over 3.2 million ([1.00-0.19] x $3,951,000$ ) cases go untreated. Even more worrying, only about a quarter of those seeking care actually take antibiotics.
\end{abstract}

Community case management (CCM) of pneumonia (4), complementing facility-based management, is a strategy to deliver antibiotics outside of health facilities where access to treatment is poor. $\mathrm{CCM}$ of pneumonia requires training community health workers (CHWs) to use algorithms developed in the 1980s (5) to assess danger signs in children with cough, count respiratory rates, and look for chest in-drawing to classify respiratory illness. Research has shown that CHWs can manage respiratory illness and prescribe antibiotics appropriately (6-9), with few exceptions $(10,11)$. A meta-analysis of nine studies found that $\mathrm{CCM}$ of pneumonia reduced overall and pneumonia-specific mortality in children $0-4$ years by $24 \%$ and $36 \%$, respectively (12). In 2002, the World Health Organization (WHO) convened experts meeting to review the evidence and field experience of CCM of pneumonia (13). A 2005 joint policy from WHO and UNICEF also recommended that "community-level treatment [of pneumonia] be carried out by well-trained and supervised CHWs" (14).

The global health community has renewed appeals for far more action to prevent and treat child pneumonia (15). Case management of pneumonia remains a central control strategy, both through facilities and in the community (15). Increasingly countries are both adopting policies for and implementing CCM (16).

Ethiopia has a promising, pro-poor strategy, the Health Extension Program (HEP), launched in 2004-5, to support its Essential Health Services Package (17). Central to the HEP are Health Extension Workers (HEW), two per kebele, who deliver "promotive, preventive and selected curative health care services in an accessible and equitable manner... with special attention to mothers and children... in rural areas" (1821). Ethiopia has nearly achieved its goal of training 30,000 HEWs (females at least 18 years old with $10^{\text {th }}$ grade education), strategically deploying them to their home kebeles. HEWs manage diarrhea with oral rehydration therapy (ORT); and malaria with Coartem ${ }^{\circledR}$. after rapid diagnostic tests. They assess, classify, and refer - but do not treat - suspected pneumonia.

\footnotetext{
${ }^{\mathrm{T}}$ Head, Health and Nutrition Unit, Save the Children USA, Addis Ababa, Ethiopia; ${ }^{2}$ Senior Child Survival Advisor, Save the Children USA, Westport, CT, USA; ${ }^{3}$ Senior Health Advisor, Save the Children, USA, Juba, Southern Sudan; ${ }^{4}$ Monitoring and Evaluation Officer, Save the Children USA, Hawassa, Ethiopia; ${ }^{5}$ Independent Consultant, Minneapolis, MN, USA; ${ }^{6}$ Africa Regional Health Advisor, Save the Children USA, Pretoria, South Africa
} 
Pneumonia control rests on pentavalent (EDPT-Hepatitis b-Hemophilus influenzae, type b).and measles vaccines and case management accessible to sick children in facilities and in communities. Introducing and scaling up these strategies will help Ethiopia get on track to achieve Millennium Development Goal 4.

Prior to the roll-out of the Health Extension Package (HEP) and wide-spread deployment of Health Extension Workers (HEWs), Save the Children USA (SC) had the opportunity to test CCM for pneumonia as well as for malaria and diarrhea, in a typical, rural, poor, subSaharan African setting, which we here report.

\section{Methods}

Project Context SC partnered with the Regional Health Bureau (RHB), zonal authorities, Woreda Health Office, and communities to deliver child survival interventions (Table 1) in Liben District (Guji Zone, Oromiya Region) through two Child Survival grants (1997-2006). The district was home to 138,000 agro-pastoralists, including 26,000 under-fives, in 37 widely dispersed rural and five urban/peri-urban kebeles, most of which lacked access to the woreda's six health facilities.

The approach in Phase 1 (1997-2001) was to: (1) train/retrain government health workers from the health facilities in IMCI; (2) support them to provide regular outreach clinics for preventive interventions; (3) establish, train and support gender-balanced Bridge-to-Health Teams (BHTs) of three existing traditional practitioners, one team per kebele, to mobilize demand for services and to provide messages for healthy household practices; and (4) form a health action committee (HAC) comprised of community leaders and BHT members to mobilize each kebele.

The approach in Phase 2 (2001-2006) was to: (1) continue the Phase 1 activities; (2) add interventions to prevent HIV/AIDS and promote maternal and newborn health; and (3) test CCM as a strategy to deliver curative interventions in 24 of the 27 most remote kebeles (three were excluded because their extreme remoteness precluded supervision).

During the program implementation period, Liben Woreda benefitted from other NGOs: Cooperazioine Internazionale (COOPI) constructed water points, Gesellschaft für Technische Zusammenarbeit (GTZ) supported education and reforestation, SOS; Sahel Ethiopia supported reforestation, and VOCA; supported animal health. None of them delivered community or facility health interventions. During the same period, 13 health posts were constructed, but these did not provide pneumonia case management.

CCM Strategy: The Regional Health Bureau gave written concurrence to pilot-test CCM (2001); woreda and zonal partners gave oral concurrence (2002); and
Federal MOH gave oral concurrence (2003) and drugs (2005). With full community participation, we selected two BHT members from each of 24 remote kebeles (with an estimated 18,150 children under five), according to: willingness, BHT or HAC membership, literacy, community respect, kebele residence, project performance, and commitment. Unfortunately only male members fulfilled the literacy requirement. The Woreda Health Office met with community members and agreed that a drug fee based on a $25 \%$ drug price was affordable and sustainable. There was no consultation fee.

Project staff, the Head of the Woreda Health Office, and FMOH Family Health Department staff visited CARE's CCM project in Siaya, Kenya (11). We adapted its comprehensive IMCI curriculum to our provider profile and priority diseases, such as, pneumonia, malaria and diarrhea. The trainer's manual contained technical background, training methods, required materials, and knowledge and skills acquired after each session. The technical heart of the approach was a simplified, fieldtested algorithm, translated into Oromiffa, for assessing, classifying and treating cough, diarrhea and fever in two age groups: young infants ( 7 days to 2 months) and children (2 to 59 months). With input from $\mathrm{FMOH}$, WHO and UNICEF child health experts, we printed registers and supervisory checklists, laminated IMCI messages, and obtained Family Health Booklets for individual and group health education by CCM workers and BHTs.

We trained (1) thirteen health workers using the 11-day IMCI course to supervise CCM workers and to ensure consistent treatment at community and facility levels and (2) three trainers using 5-day facilitation training. They trained 45 trainees (three per trainer) using the WHO 11day (later reduced to 7 days, including 1.5 days for revolving drug fund and reporting) IMCI course, through lectures, role-plays, drills, video with case studies, and clinical practice during a one-day visit to two communities where ill children were identified and treated in schools or under shade-trees. Each trainee managed at least five cases of fever, Acute Respiratory Infection (ARI), and diarrhea. Training was provided in Oromiffa, including a voice-over of the English-language WHO IMCI video.

We assessed knowledge and skills through post-training written and practical tests. We accredited trainees achieving $60 \%$ to provide CCM services from their home and at outreach clinics. We chose a lower cut-off than used in IMNCI training for doctors and nurses (80\%) because CCM workers had little education (class four to nine) or work experience requiring literacy. Each CCM worker received a drug cupboard, bag, register, reporting format, IEC materials, and drugs: tablets of cotrimoxazole, chloroquine, and sulfadoxinepyrimethamine (Fansidar); tetracycline eye ointment; ORS; paracetamol; vitamin A; and clean gloves. The five 
trainees who scored below $60 \%$ continued regular health education activities. We provided a three-day refresher training six months after the first training because CCM workers were unlikely to maintain unused skills, given unexpected additional delays in pilot-test approval.

Health facility, Woreda Health Office and Project personnel supervised the outreach clinics monthly. They monitored skills using direct observation of cases (or reported management of simulated cases) and reviewed registers. $\mathrm{CCM}$ workers reported cases treated by diagnosis, referrals, and stock. Neither births nor deaths were tracked. Supervisors aggregated the information and submitted it to the Woreda Health Office and the project office.

Evaluation We used eight methods to evaluate the experience, all in August 2006, except for the household surveys. (1) We reviewed all CCM workers' registers to characterize their service; (2) reviewed all $\mathrm{MOH}$ facility treatment registers to quantify case management for under-fives; (3) reviewed project records; (4) individually tested the 40 accredited CCM workers by three simulated cases (one each for cough, diarrhea, and fever); (5) reviewed health facility supervision records of CCM workers, specifically examining pneumonia case management; (6) conducted three district-wide household surveys; (7) conducted in-depth interviews with several wored $a$ and health facility staff and HAC members; and (8) conducted six focus group discussions: one with seven CCM workers and five with a total of 45 mothers.

The household surveys measured mothers' (of children $<24$ months) knowledge and reported practices: at baseline of Phase 1 (August 1997 [n=369]), at end-line of Phase 1 which was baseline of Phase 2 (July-August $2001[n=360]$ ), and at end-line of Phase 3 (June 2006 $[\mathrm{n}=114])$. We used a 30-cluster, multi-stage random sample for the first two and lot quality assurance simple random sample for the third.
Analysis: We used Excel to analyze CCM workers' services and hand-tallies to score their service quality. For the latter we assessed the worker's ability to determine patient age; ask screening questions, danger signs, and assessment questions; classify disease; treat; and counsel in each of three scenarios. We also determined his knowledge of respiratory rate cut-offs by age group, general danger signs, and response to general danger signs. We calculated scores weighting each component equally.

We determined the expected pneumonia cases by summing the following for all 24 kebeles: $\{$ (the duration [in years] the kebele was served by a CCM worker) $\mathrm{x}$ (child population of the kebele) $\mathrm{x}$ ( 0.3 cases per child per year)\} (1). We calculated the "pneumonia treatment rate" by dividing the actual cases treated by the expected cases.

We present findings through a results framework (22) which hypothesizes that lives are saved through increased use of evidence-based interventions and that use increases as supply (i.e., access and quality) of and demand (e.g., knowledge of danger signs and sources of care) for interventions increases.

\section{Results}

Use of CCM Services CCM Worker Register Review:Five of $38 \mathrm{CCM}$ workers forgot their registers on evaluation day, and one register was incomplete, leaving 32 registers to analyze (representing 22 of 24 kebeles). Most CCM workers (72\% [23/32]) had been active for 12-13 months. They saw 4787 cases (average: 150 cases/worker; ranged from 11-496), of which $41(<1 \%)$ were referred. The 32 workers contributed 362 personmonths of service (average: 13 cases per worker per month; ranged from 2-38).

Table 1: Interventions by Delivery Strategies: Phase 1 (Oct 1997-Sep 2001) and Phase 2 (Oct 2001- Sep 2006) (BHT=Bridge-to-Health Team, CCMW=Community Case Management Worker)

\begin{tabular}{|c|c|c|c|c|}
\hline \multirow[t]{2}{*}{ Interventions } & \multicolumn{4}{|c|}{ Delivery Strategies by Phase (1 and/or 2 ) } \\
\hline & Facility & Outreach & BHT & CCMW \\
\hline Exclusive breastfeeding & 1 and 2 & & 1 and 2 & \\
\hline Complementary feeding & 1 and 2 & & 1 and 2 & \\
\hline Vitamin A supplementation & 1 and 2 & 1 and 2 & & \\
\hline Antenatal care & 1 and 2 & 1 and 2 & & \\
\hline Family planning & 2 & 2 & & \\
\hline Clean delivery & 1 and 2 & & 1 and 2 & \\
\hline Essential newborn care & 1 and 2 & & 1 and 2 & \\
\hline Immunizations & 1 and 2 & 1 and 2 & & \\
\hline ORS & 1 and 2 & & 1 and 2 & 2 \\
\hline Antibiotic for pneumonia & 1 and 2 & & & 2 \\
\hline Antibiotic for dysentery & 1 and 2 & & & 2 \\
\hline Antibiotic for sepsis & 1 and 2 & & & \\
\hline Anti-malarial & 1 and 2 & & & 2 \\
\hline Condom & 2 & 2 & 2 & \\
\hline
\end{tabular}


CCM cases involved four syndromes: fever (38\%), ARI $(30 \%)$, diarrhea $(19 \%)$, and conjunctivitis (14\%) (Table $2)$. The most common classifications were: malaria $(36 \%)$, pneumonia $(26 \%)$, conjunctivitis $(14 \%)$, and acute watery diarrhea with some dehydration (12\%). CCM workers reported no severe dehydration. Bloody diarrhea was common (24\%), but five kebeles accounted for $75 \%$ of cases, consistent with local outbreaks. Young infants contributed few cases $(<1 \%)$, probably because cultural norms confined mothers and infants at home for two months postpartum.

Table 2: Cases Treated by CCM Workers by Diagnosis and Age (July 2005-August 2006)*

\begin{tabular}{llll}
\hline Diagnosis & Age Group & & Total \\
& $\mathbf{0 - 1}$ & $\mathbf{2 - 5 9}$ months & \\
\hline month & 0 & 147 & 147 \\
Pne Pneumonia ("cough or cold") & 9 & 1242 & 1251 \\
Severe Pneumonia Nery Severe Disease & 5 & 28 & 33 \\
TOTAL: ARI & 14 & 1417 & 1431 \\
Acute Watery diarrhea no dehydration & 1 & 73 & 74 \\
Acute Watery diarrhea : some dehydration & 1 & 558 & 559 \\
Acute Watery diarrhea : severe dehydration & 0 & 0 & 20 \\
Persistent diarrhea & 1 & 19 & 210 \\
Bloody diarrhea & 0 & 210 & 27 \\
Very Severe Disease & 5 & 22 & 890 \\
TOTAL: Diarrhea & 8 & 882 & 1713 \\
Malaria & 0 & 1713 & 47 \\
Measles & 0 & 47 & 3 \\
Complicated Measles & 0 & 3 & 38 \\
Very Severe Disease & 2 & 36 & 1801 \\
TOTAL: Fever & 2 & 1799 & 666 \\
Conjunctivitis & 1 & 665 & 4787 \\
GRAND TOTAL & 24 & 4763 & \\
\hline
\end{tabular}

*Source: CCM workers' registers

MOH Facility Register Review: During the same period, all the 19 health facilities (13 health posts, which do not provide pneumonia treatment, were added during the CCM pilot), including the district hospital, saw 1944 cases of ARI, diarrhea and fever in under-fives. CCM workers saw 2.5 times as many patients with these diagnoses as health facility staff. Applying the activity level of the $32 \mathrm{CCM}$ workers for whom we have data to the six whose registers were not analyzed, yielded 5684 $(4787 \times 38 / 32)$ likely cases treated, nearly three-fold (2.9) the load of their facility-based counterparts.

CCM workers treated one third (34\% [1284/3724]) of the expected pneumonia cases, perhaps a slight overestimated since the "pneumonia months," June to August ( $25 \%$ of a year), were somewhat over-represented $(32 \%$ [116/362]) in the CCM service months.

Availability of CCM Services Project Record Review: The CCM strategy increased access to case management (kebeles with $\mathrm{MOH}$ facility or CCM workers: 19\% in 2001 to $65 \%$ in 2006). SC trained 45 and accredited 40 CCM workers, two of whom ceased functioning. Their attrition rate was one tenth that of IMCI-trained health facility staff (5\% [2/40] vs. 50\% [7/14]) during the same period.

SC aimed to provide one or usually two CCM workers in the 24 kebeles (average total population/kebele 4048; range: 2253-7974). At end-line, after 13 months of CCM implementation, 14 kebeles had two workers and 10 had one worker. On average, a CCM worker covered a total population of 2736 (range: 1293-6554) or an estimated 492 children (range: 233-1180.)

Quality of CCM Services Simulated Cases: The CCM workers provided good quality care. During the final evaluation most workers (32/40) scored above $80 \%$, and more than half (24/32) scored above $90 \%$ on tests of their ability to apply standard case management to three different diseases.

Supervision Record Review: Monitoring data also confirmed good quality. Supervisors tracked pneumonia case management since this was a project result. Supervisors' records of CCM register review showed that nearly all the 1212 pneumonia cases monitored were completely assessed (97\%), consistently classified according to assessment findings (97\%), and consistently treated according to classification (96\%). Monitoring data also confirmed availability of cotrimoxazole (no stock-out in previous month: 100\% [45/45]). CCM workers reported their activities reliably (report filed in the last quarter: $100 \%$ [45/45]).

Demand for CCM Services/Household Survey: Demand includes knowledge ("case detection") and careseeking. Care-givers' knowledge of fast or difficult breathing as signs of pneumonia increased from $39 \%$ to 92\% between 1997 and 2006, and nearly all mothers 
(94\%) knew at least two childhood danger signs by 2006. Appropriate care-seeking for the three syndromes increased over the project (Table 3). Specifically careseeking for cough and difficult or rapid breathing increased from $30 \%$ to $54 \%$ in Phase 1 when communities were trained to recognize danger signs and seek care at health facilities. The level further increased in Phase 2 to $84 \%$ when CCM workers were deployed. We know that all the increase (Figure 1) was due to CCM because surveyed mothers identified sources of care and because health posts did not provide pneumonia case management. Focus Group Discussion: Mothers at endline knew their kebele's CCM worker, the conditions he treated, and his activities.

Table 3: Care-Seeking by Syndrome and Year [\% (n/d) ${ }^{*}$

\begin{tabular}{llll}
\hline Syndrome & 1997 & $\mathbf{2 0 0 1}$ & $\mathbf{2 0 0 6}$ \\
\hline ARI & $30.1(25 / 83)$ & $57.9(33 / 57)$ & $84.0(79 / 94)^{\star *}$ \\
Diarrhea & $43.6(48 / 110)$ & $63.8(29 / 80)$ & $93.8(106 / 113)^{\star *}$ \\
Fever & $28.6(14 / 49)$ & $67.6(23 / 34)$ & Not asked \\
\hline
\end{tabular}

*Source: population-based household surveys

${ }^{* *}$ weighted values are $83 \%$ and $93 \%$.

Enabling Environment for CCM During In-depth Interviews:- The kebeles' HACs and health facility staff supported the CCM strategy. Managing drugs remained a challenge, though, because the $\mathrm{MOH}$ required detailed documentation (vouchers for receipt and each sale). Managing cash proved equally challenging, but we noted no theft, and most users were able to pay, consistent with the initial WHO-community price-setting dialogue. The brief implementation period precluded simplifying and testing. The policy environment remained opposed to CCM for pneumonia.
CCM Workers Speak (Focus Group Discussion): Seven CCM workers from Boba, Siminto, Raro and Sokora volunteered because child health was such a "deep-rooted problem" in their communities and because of the benefits of the training. Accomplishments included: accessible case management at reduced cost, increased community demand for care with less use of "illegal drug vendors," and community recognition. Although they identified no important knowledge gaps, they recommended refresher training, more CCM workers, and a furnished separate space to provide service.

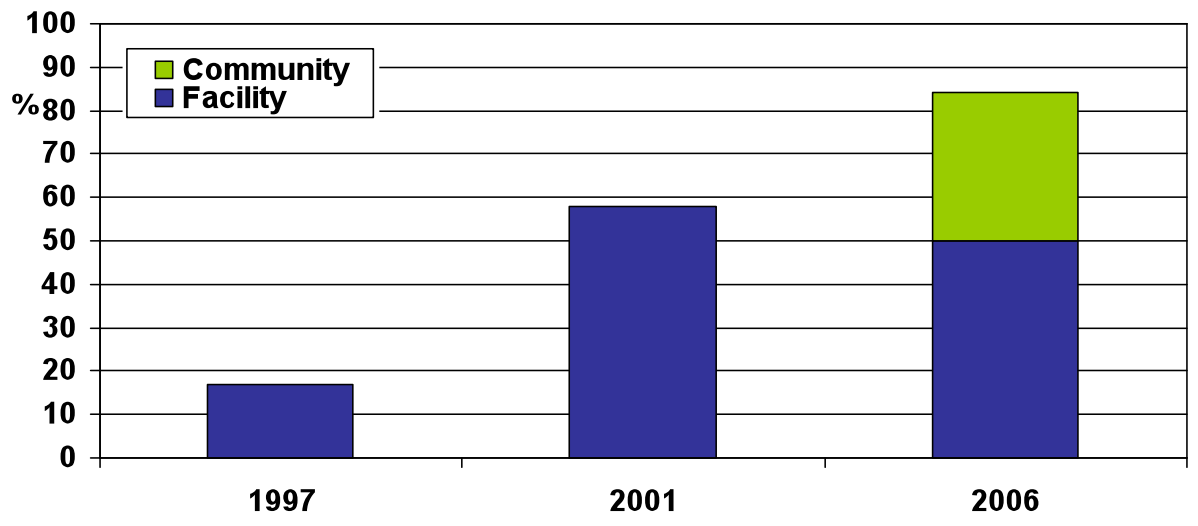

*Source: population-based household surveys

Figure 1: Care-Seeking for cough and difficult or fast breathing by source of case management: Liben Woreda (1997-2006)*

\section{Discussion}

The availability of, quality of, demand for, and use of CCM were high and more remarkable since this was the first year of implementation. The new and complex strategies whose implementers lack a strong educational background often need 12-18 months to function smoothly. Although the implementation never matured, the strategy temporarily filled a service gap in remote kebeles of Liben Woreda. Although we lack the proof, we suspect that lives were saved by bringing case management to highly vulnerable, isolated, poor children. CCM was valued by beneficiaries, providers, and local $\mathrm{MOH}$ partners. CCM worker retention was high, but the observation period was brief. 
This "natural experiment" of phased programming illustrates the effect and limitation of: (1) community mobilization and health education for facility-based pneumonia case management (phase 1: 1997-2001) and (2) the added benefit of community-based care after demand generation (phase 2: 2006). The figure suggests the answer to the question of what would have happened without CCM: care-seeking at health facilities would have remained static at about $50-60 \%$. On the other hand, if the 13 new health posts had provided pneumonia case management, facility-based care-seeking surely would have increased.

The case spectrum is open to interpretation. Assuming correct classification by CCM workers: (1) the low levels of minor disease ("no pneumonia") suggested that CCM services were not overused; (2) the low levels of very severe disease $(2 \%)$ and the absence of severe dehydration suggested early and appropriate careseeking; and (3) the low rates of referral are consistent with successful CCM treatment. The widespread community knowledge of danger signs and sources of care support this interpretation. On the other hand, some mis-classification is likely, and perhaps (1) some "no pneumonia" was classified as "pneumonia," and (2) all severe dehydration was missed, but we do not know its true incidence. Moreover, the low referral rate may have simply underscored the non-feasibility of reaching a facility even with a desperately ill child. After all, lack of access was the rationale for the strategy in the first place.

Remaining challenges include: (1) understanding the low referral rate (non-referral vs. refusal), the wide variation in CCM worker treatment levels, and the age-structure of cases; (2) designing a practical information system to track drugs; (3) testing an appropriate cost-recovery strategy in which the benefit justifies the effort, (4) testing a strategy for the most inaccessible communities where supervision is not feasible; and (6) continuing policy dialogue, aiming to permit and ultimately to mandate CCM for inaccessible areas where supervision is possible.

Since our pilot-test, Ethiopia has deployed thousands of HEWs, who are far better educated and trained than the CCM workers of Liben. Preventive interventions are more cost-effective than curative ones in the long run. But before achieving the "long run," a judicious choice of curative interventions (23) - including accessible pneumonia case management - will reduce suffering and death. HEWs delivering pneumonia treatment makes sense. One could argue that what they already do (complicated Rapid Diagnostic Tests for fever; assess and classify ARI) is more complex than what they cannot do: treat pneumonia. Moreover, they dispense Coartem, which is ten times as costly as cotrimoxazole.

Put another way, a typical kebele has about 1000 children and 300 cases of child pneumonia annually. In other words, the HEWs from one kebele might refer suspected pneumonia almost daily. Many referrals are not feasible due to health center inaccessibility. Non-feasible recommendations can jeopardize HEW credibility. On the other hand, treating pneumonia can enhance credibility. In addition, many sick children have signs of both malaria (fever) and pneumonia (rapid breathing), and deserve treatment for both. Treating one disease (malaria) at a health post and referring a common comorbidity (pneumonia) to a health center may not be the best strategy.

Recent studies shed light on HEW training (19), continuing education (20), and working conditions (21). Not surprisingly, the first intake of such an ambitious program had challenges. On balance, the HEWs were and are highly valued by communities - even if their training, supervision, role in the $\mathrm{MOH}$ team, career-ladder options, and planned commitment need review. One observation seems especially relevant to role, job satisfaction, and perhaps commitment. Teklehaimanot reported that "curative care was a pervasive request in almost all communities" because of distance and cost of travel (21). Indeed, the just concluded mid-term review of the $3^{\text {rd }}$ Health Sector Development Programme stated that "sustaining the trend of UFMR $[<5$ mortality rate] reduction is unlikely unless community-based pneumonia management is introduced..." (24).

Based on need, existing HEW strategy, and global experience including ours, we recommend that Ethiopia: (1) train, equip and supply HEWs to deliver pneumonia treatment to children, (2) test small-scale, cost-recovery schemes to assure sustainability, and (3) consider a scaled-back (less training, less education, fewer duties) interim package for communities with the following characteristics: limited human resources, extreme remoteness, small or mobile population, and insecurity among others.

\section{Acknowledgements}

This project was supported by the United States Agency of International Development (USAID) through two Cooperative Agreements (FAO-A-97-00054-00 and FAO-A-00-97-00054-00).

\section{References}

1. Rudan I, Boschi-Pinto C, Biloglav Z, Mulholland K and Campbell H. Epidemiology and etiology of childhood pneumonia, Bulletin of World Health Organization 2008;86(5):408-415.

2. UNICEF/WHO, Pneumonia the forgotten killer of children, 2006.

3. Central Statistical Agency [Ethiopia] and ORC Macro. 2006. Calverton Maryland Ethiopia Demographic and Health Survey 2005. Addis Ababa, Ethiopia and Calverton, Maryland, USA: Central Statistical Agency and ORC Macro.

Ethiop. J. Health Dev. 2009;23(2) 
4. Dawson P., Houston, RM, Karki S, Thapa S. Management of childhood pneumonia: Improved treatment using community-based approaches in Nepal. (Unpublished manuscript).

5. Shann F, Hart K, Thomas D. Acute lower respiratory tract infections in children: possible criteria for selection of patients for antibiotic therapy and hospital admission. Bull World Health Organization 1984;62(5):749-53.

6. Hadi A. Diagnosis of pneumonia by community health volunteers: experience of BRAC, Bangladesh. Trop Doct 2001; 31 (2): 75-7.

7. Charleston R, Johnson L, Tam L. CHWs trained in ARI management. Sante Salud 1994;4:14.

8. Mehnaz A, Billoo AG, Yasmeen T. Nankani K. Detection and management of pneumonia by community health workers -a community intervention study in Rehri village, Pakistan. J Pak Med Assoc. 1997;47(2):42-5.

9. WHO. Case management of acute respiratory infections in children: Intervention studies. Geneva, WHO/ARI/88.2, 1988, p. 31.

10. Rowe SY, Kelly JM, Olewe MA, Kleinbaum DG, McGowan JE, Jr, McFarland DA. et al. Effect of multiple interventions on community health workers' adherence to clinical guidelines in Siaya district, Kenya. Trans R Soc Trop Med Hyg 2007;101(2):188-202.

11. Kelly JM, Osamba B, Garg RM, Hamel MJ, Lewis JJ, Rowe SY et al. Community health worker performance in the management of multiple childhood illnesses: Siaya District, Kenya, 19972001. Am J Public Health 2001;91 (10):1617-24.

12. Sazawal S, Black RE. Effect of pneumonia case management on mortality in neonates, infants, and preschool children: a meta-analysis of communitybased trials. Lancet 2003;3(9):547-56.

13. WHO. Meeting Report: Evidence base for community management of pneumonia, Stockholm June 11-12. WHO/FCH/CAH/02.23. Geneva, World Health Organization, 2002.

14. WHO/UNICEF. Joint Statement: Management of Pneumonia in Community Settings. Geneva and New York, World Health Organization and UNICEF, 2004.
15. UNICEF/WHO. Pneumonia: the forgotten killer of children. New York and Geneva, UNICEF and World Health Organization, 2006.

16. Marsh D, Gilroy K, Van de Weerdt R, Wansi E, Qazi S. Community case management of pneumonia - at a tipping point?, Bulletin of World Health Organization, 2008;86(5)381-389.

17. Federal Ministry of Health. Essential Health Services Package for Ethiopia, 2005.

18. Federal Ministry of Health [Addis Ababa, Ethiopia]. Health Extension Program in Ethiopia - Profile. Health Extension and Education Center, June 2007.

19. Kitaw Y, Ye-Ebiyo Y, Said A, Desta H, Teklehaimanot A. Assessment of the training of the first intake of health extension workers. Ethiop. J. Health Dev. 2007;21(3):232-239.

20. Ye-Ebiyo Y, Kitaw Y, G/Yohannes A, Girma S, Desta H, Seyoum A, Teklehaimanot A. Study on health extension workers: Access to information, continuing education and reference materials. Ethiop. J. Health Dev. 2007;21(3):240-245.

21. Teklehaimanot A, Kitaw Y, G/Yohannes A, Girma S, Seyoum A, Desta H, Ye-Ebiyo Y. Study of the working conditions of health extension workers in Ethiopia. Ethiop. J. Health Dev. 2007;21(3):246-259.

22. Marsh DR, Alegre JC, Waltensperger KZ. A results framework serves both program design and delivery of services. Journal of Nutrition 2008;138 630-633.

23. Jones G, Steketee RW, Black RE, Bhutta ZA, Morris SS, and the Bellagio Child Survival Study Group, How many child deaths can we prevent this year? Lancet 2003;362: 11-17.

24. Chabot J, Maalim A, Kampo A, O'Connell A, Herforth A, Tesfahun A, et al., Ethiopia Health Sector Development Programme (HSDP III), $2005 / 06-2010 / 11$ Mid-Term Review, $05^{\text {th }}$ May $-5^{\text {th }}$ June 2008. Addis Ababa, $12^{\text {th }}$ July 2008. 